\title{
Calculation of Electric Field Gradients in Isolated Molecules Using the FPLAPW-Code WIEN95*
}

\author{
Torsten Soldner**, Wolfgang Tröger, Tilman Butz, Peter Blaha ${ }^{a}$, and Karlheinz Schwarz ${ }^{a}$ \\ Abteilung Nukleare Festkörperphysik, Universität Leipzig, Linnéstraße 5, D-04103 Leipzig \\ a Institut für Technische Elektrochemie, Technische Universität Wien, Getreidemarkt 9/158, \\ A-1030 Wien \\ Z. Naturforsch. 53a, 411-418 (1998); received December 31, 1997

\begin{abstract}
The full potential linearized augmented plane wave method as embodied in the program package WIEN95 was originally developed for crystalline solids and is based on crystal periodicity. The present work demonstrates that it is applicable to isolated molecules with the examples of $\mathrm{CdCl}_{2}, \mathrm{HgF}_{2}$, and $\mathrm{HgCl}_{2}$ by investigating the required size of artificially enlarged unit cells and by calculating bond distances and vibrational frequencies in excellent agreement with experimental data. The dependence of electric field gradients at $\mathrm{Cd}$ and $\mathrm{Hg}$, respectively, on bond angle is investigated. A point charge like behaviour of energetically low lying states is found, whereas large discrepancies from the point charge model occurred for the energetically high lying valence states.
\end{abstract}

\section{Introduction}

In the past years, the full potential linearized augmented plane wave (FPLAPW) method as embodied in the program package WIEN95 [1] has proven to be an accurate and powerful method to calculate electron densities and electric field gradients (EFG) in crystals [2]. For molecules, this method is not directly applicable since it requires crystal periodicity. On the other hand, most methods designed to calculate molecules are specialized in describing the chemical bonding (in particular for lighter elements) and therefore suffer from a poor basis set near the nuclei and are thus not applicable to heavy nuclei. Nevertheless, Hemmingsen and Ryde [3] calculated the EFG at $\mathrm{Cd}$ in $\mathrm{CdCl}_{2}$ using the Hartree-Fockmethod including some correlation corrections, but no experimental or competing theoretical data are available to check these results.

We investigated the possibility to apply WIEN95 to isolated molecules. By comparing measurable properties (bond distances, vibrational frequencies) with the calculated values we can test the quality of such calculations ${ }^{1}$.

\footnotetext{
* Presented at the XIVth International Symposium on Nuclear Quadrupole Interactions, Pisa, July 20-25, 1997.

** Present address: Physik-Department E21, Technische Universität München, James-Franck-Straße, D-85748 Garching.

1 To our knowledge, there are no experimental data on the EFG in isolated molecules of $\mathrm{CdCl}_{2}, \mathrm{HgF}_{2}$, and $\mathrm{HgCl}_{2}$.
}

Reprint requests to Prof. T. Butz; Fax: 0341-9732748.
Encouraging results would allow us to calculate EFGs in molecules, too, with the same method as successfully used for solids. Comparisons to other methods applicable to molecules only would be possible. In isolated molecules, effects from the crystal structure are not superimposed on the contributions from different electronic states, influences of bond angles and distances. Therefore in a free molecule the origin of the EFG could be understood more easily than in solids and common rules could possibly be extracted.

We compare our results with EFG measurements in crystals and other calculations (results of Fraenzke and Butz [4], Tröger et al. [5], and Hemmingsen and Ryde [3]).

\section{Method}

The FPLAPW method divides the unit cell of a crystal into spheres around the atoms and an interstitial region. The wave functions of the valence states at a $\boldsymbol{k}$-point in the first Brillouin zone are expanded in spherical harmonics within the spheres and in plane waves in the interstitial region:

$$
\varphi_{\boldsymbol{k}}(\boldsymbol{r})=\left\{\begin{array}{rr}
\Sigma_{l m}\left(A_{l m}^{\beta, \boldsymbol{k}} u_{l}^{\beta}\left(r, E_{l}^{\beta}\right)\right. \\
\left.+B_{l m}^{\beta, \boldsymbol{k}} \dot{u}_{l}^{\beta}\left(r, E_{l}^{\beta}\right)\right) Y_{l m}(\boldsymbol{r}) \\
\text { sphere of atom } \beta \\
\frac{e^{i \boldsymbol{k} \boldsymbol{r}}}{\sqrt{\Omega}} \Sigma_{\boldsymbol{K}} u_{\boldsymbol{K}}^{\boldsymbol{k}} e^{i \boldsymbol{K} \boldsymbol{r}} & \text { interstitial region . }
\end{array}\right.
$$


$l$ and $m$ are the angular momentum quantum numbers, $u_{l}^{\beta}\left(r, E_{l}^{\beta}\right)$ is the (numerical) solution of the radial Schrödinger equation within the sphere $\beta$ at energy $E_{l}^{\beta}$, $\dot{u}_{l}^{\beta}$ is its energy derivative, $\boldsymbol{K}$ runs over the vectors of the reciprocal lattice, and the $u_{\boldsymbol{K}}^{\boldsymbol{k}}$ are the plane wave coefficients. For each $\boldsymbol{k}$-point these coefficients have to be calculated by diagonalizing a matrix with dimensions equal to the number of $\boldsymbol{K}$ vectors. Therefore, the plane wave expansion is severely limited by the available size of the computer main memory.

The core states (in this work: $\mathrm{F} 1 \mathrm{~s}, \mathrm{Cl} 1 \mathrm{~s}$ to $2 \mathrm{p}, \mathrm{Cd} 1 \mathrm{~s}$ to $4 \mathrm{~s}$, and $\mathrm{Hg} 1 \mathrm{~s}$ to $5 \mathrm{~s}$ ) are treated as spherically symmetric. The influence of $\mathrm{Hg} 4 \mathrm{f}$ states on the EFG was tested by treating them as valence states and found to be in the order of $1 \%$. Therefore, these states were treated as core states in further calculations.

Full point group symmetry was used to decrease the computational effort.

To apply the FPLAPW method to molecules it is necessary to construct a large supercell which contains one molecule. This supercell is repeated periodically in all three directions. Only one $\boldsymbol{k}$-point $\boldsymbol{k}=(0,0,0)$ is needed, and the unit cell dimensions must be chosen sufficiently large that interactions between atoms from neighbouring cells are negligible. This can be tested by increasing the unit cell dimensions and checking the convergence of some calculated properties. We used a tetragonal unit cell (dimension $a \times b \times b)$ with the molecule axis along the $x$-direction (coordinates $(0,0,0)$ for the metal atom and $( \pm x, 0,0)$ for the halogen atom, see Figure 1). To use the space of the unit cell as efficiently as possible and to preserve the (100) mirror plane as symmetry element while bending the molecules (Section 5.2) we placed the halogen atoms at $\left( \pm R_{\mathrm{X}-\mathrm{Y}} / a \cos \Theta, R_{\mathrm{X}-\mathrm{Y}} /(\sqrt{2} b) \sin \Theta\right.$, $\left.R_{\mathrm{X}-\mathrm{Y}} /(\sqrt{2} b) \sin \Theta\right)$ with $\Theta=\pi-\theta / 2, \theta$ being the bond angle and $R_{\mathrm{X}-\mathrm{Y}}$ the bond distance between the atoms $\mathrm{X}$ and $\mathrm{Y}$.

All calculations were performed using the generalized gradient approximation (GGA) [6].

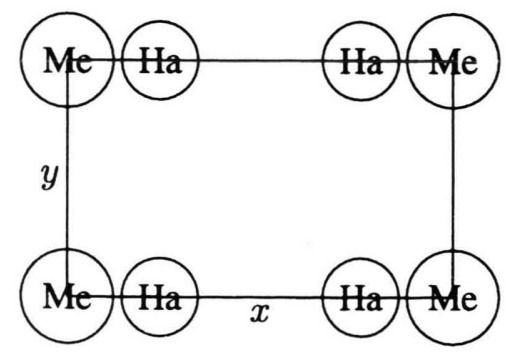

Fig. 1. Geometry of the unit cell.

\section{Test of Basis Set}

The application of the FPLAPW method to molecules requires extended tests of basis sets.

The quality of the plane wave basis set can be characterized by the parameter $R K_{\max }$ defined as

$$
R K_{\max }:=\min _{\beta}\left\{R_{\beta}\right\} \max _{\boldsymbol{K}}\{|\boldsymbol{K}|\},
$$

where $R_{\beta}$ is the radius of sphere $\beta$. Obviously, the basis set improves by increasing $R K_{\max }$ but the parameter range is restricted by main memory limitations (see Section 2).
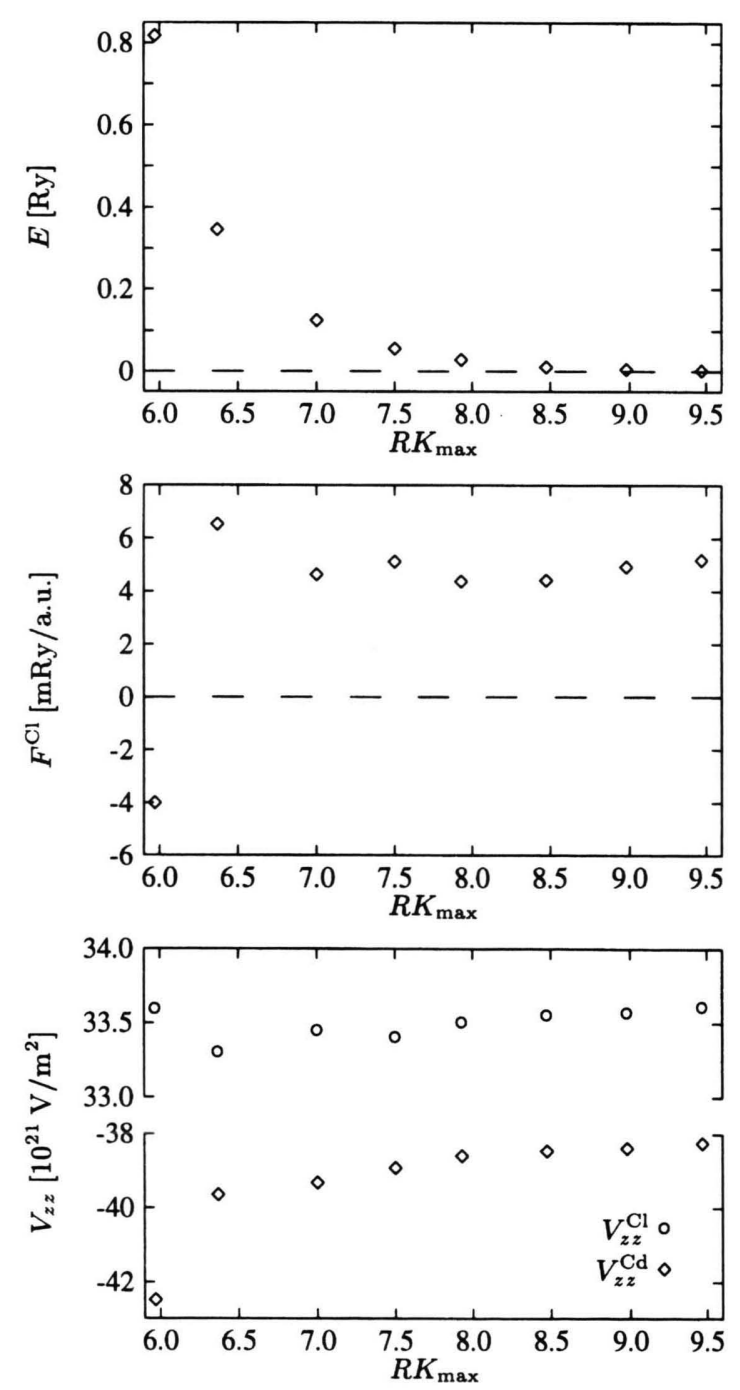

Fig. 2. Total energy $E$ (with arbitrary zero), force on $\mathrm{Cl}$ atom $F^{\mathrm{Cl}}$, and $V_{z z}$-values versus convergence parameter $R K_{\max }$ for $\mathrm{CdCl}_{2}$. 
In Fig. 2, the influence of the plane wave basis set on the total energy, the force on the $\mathrm{Cl}$ atom, and $V_{z z}$ at the $\mathrm{Cd}$ and the $\mathrm{Cl}$ nuclei are plotted for $\mathrm{CdCl}_{2}$ with $a=16$ a.u. ${ }^{2}, b=8$ a.u.. and $R_{\mathrm{Cd}-\mathrm{Cl}}=4.16$ a.u. Convergence could be reached for $R K_{\max } \geq 7.5$. Due to the large computing time and main memory requirements all further calculations were performed using a slightly smaller $R K_{\max }\left(R K_{\max }=7.0\right)$, which saves computer time but is still sufficiently accurate.

Furthermore, we tested the WIEN95-specific parameters NPT and R0 defining the radial mesh within the spheres, on which $u_{l}^{\beta}$ is calculated [7]. As expected, all these parameters have only minor influence on the results and thus are not reported here (see [8]).

\section{Test of Unit Cell Dimensions}

For $\mathrm{HgCl}_{2}$ the convergence of molecular properties versus unit cell dimensions was tested. Holding $b$ fixed

${ }^{2}$ Within this paper atomic units are used: 1 a.u. $=5.29177$. $10^{-11} \mathrm{~m}$.

\section{Total energy:}
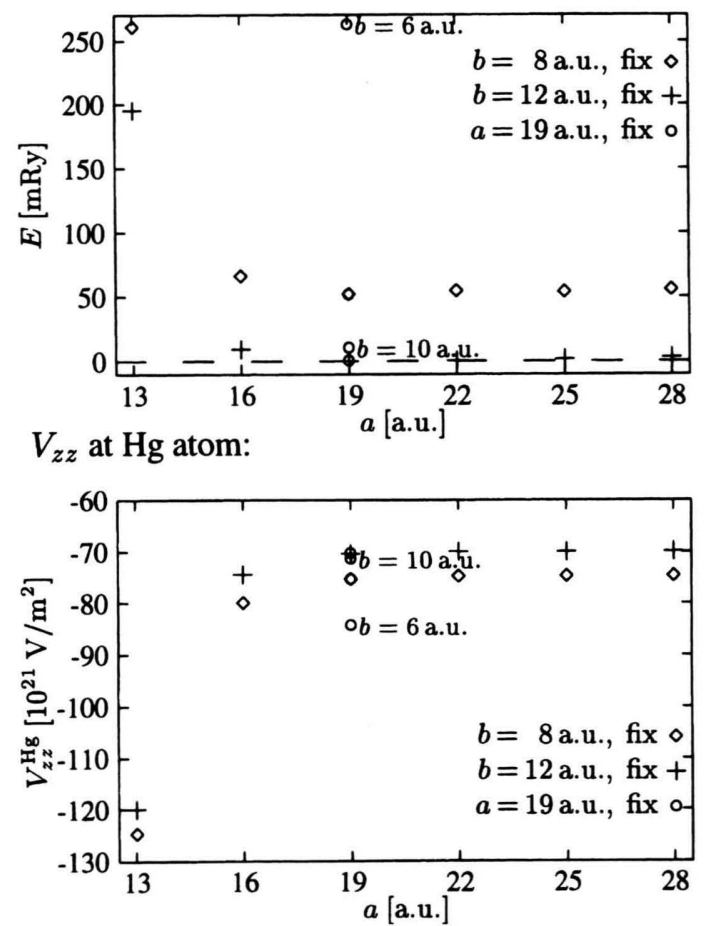

at $b=8$ a.u. and $b=12$ a.u., respectively, $a$ was varied from $a=13$ a.u. to $a=28$ a.u. while the bond distance $R_{\mathrm{Hg}-\mathrm{Cl}}$ was kept constant $\left(R_{\mathrm{Hg}-\mathrm{Cl}}=4.24\right.$ a.u. $)$. Subsequently, for $a=19$ a.u., $b$ was varied from $b=6$ a.u. to $b=12$ a.u. The results are plotted in Figure 3.

Convergence was reached for $a \geq 19$ a.u. and $b \geq$ 10 a.u. For further calculations $a=20$ a.u. and $b=12$ a.u. was used. $b$ was increased more than $a$ because we wanted to bend the molecules. Since $\mathrm{HgCl}_{2}$ contains the largest atoms of the investigated halogenides, we used these unit cell dimensions for all three compounds.

\section{Calculations of Properties of Isolated Molecules}

Using the unit cell dimensions described in Sect. 4 we calculated total energies, forces, and electric field gradients as functions of bond distance and bond angle.

\subsection{Bond Distances}

The bond distances $R_{\mathrm{X}-\mathrm{Y}}$ were varied in the range from 4.12 a.u. to 4.32 a.u. $\left(\mathrm{CdCl}_{2}\right)$, from 3.60 a.u. to 3.70 a.u.

Force at $\mathrm{Cl}$ atom:

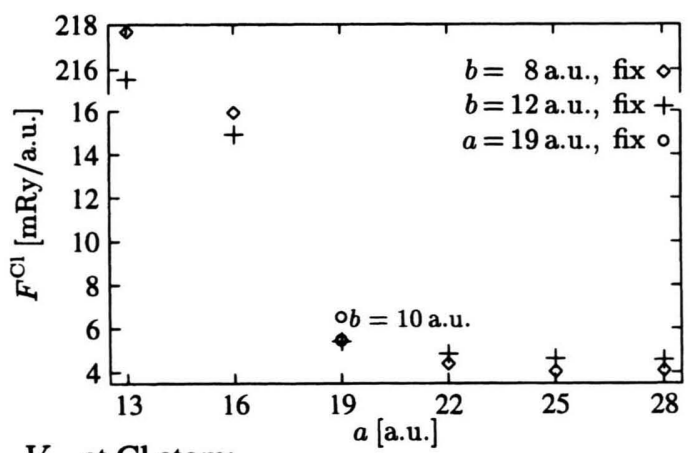

$V_{z z}$ at $\mathrm{Cl}$ atom:

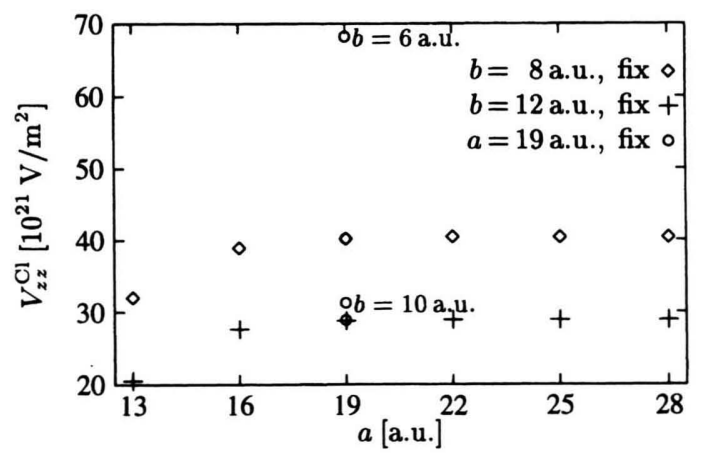

Fig. 3. Total energy $E$ (with arbitrary zero), force at $\mathrm{Cl}$ atom $F^{\mathrm{Cl}}$, and $V_{z z}$-values versus unit cell dimensions for $\mathrm{HgCl}_{2} . \mathrm{For}_{\mathrm{f}}$ this calculation, $R K_{\max }=8.0$ was used. 
$\left(\mathrm{HgF}_{2}\right)$, and from 4.20 a.u. to 4.40 a.u. $\left(\mathrm{HgCl}_{2}\right)$, respectively. The results are plotted in Figure 4.

The total energies show a nearly quadratic dependence on $R_{\mathrm{X}-\mathrm{Y}}$ and consequently the forces (defined as $F_{\mathrm{X}}=-\delta E / \delta R_{\mathrm{X}}$, where $R_{\mathrm{X}}$ is the position of atom $\mathrm{X}$, but calculated directly using the wave functions at each geometry [7]) show only small deviations from linearity. The equilibrium bond distances were determined by both minimizing total energies and adjusting for vanishing forces. The results are summarized in Table 1 and compared with experimental values from electron diffraction. A quadratic fit of the total energy dependence on $R_{\mathrm{X}-\mathrm{Y}}$ allowed to determine the force constants leading to the stretch vibration frequencies given in Table 1 , in good agreement with the experimental results from [9, 10].

\section{$\mathrm{CdCl}_{2}$ :}
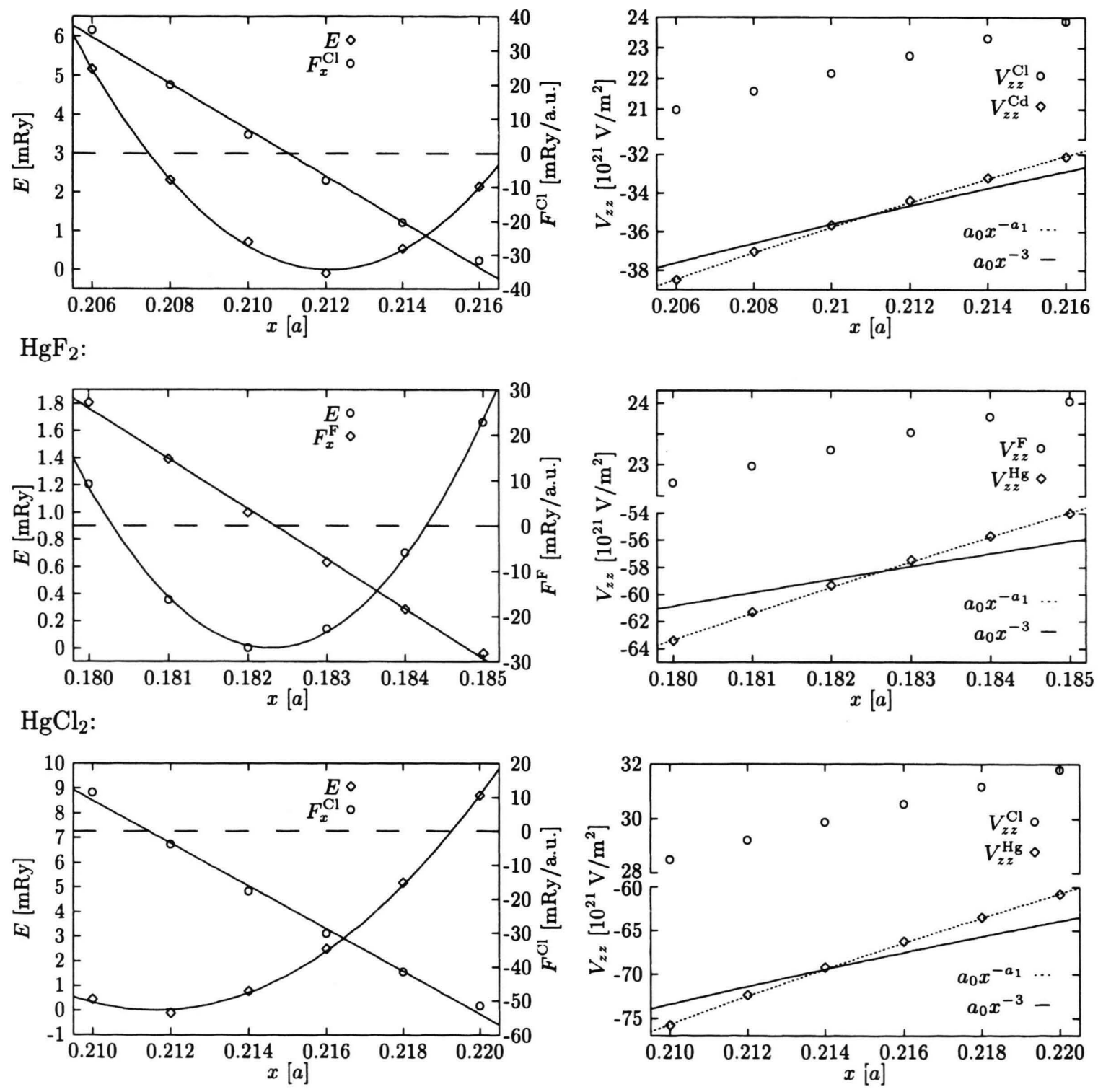

Fig. 4. Total energy $E$ (with arbitrary zero), force at halogen atom $F^{\mathrm{Y}}$, and $V_{z z}$-values versus bond distances (in units of the lattice constant $a=20 \mathrm{a}$. u.) for $\mathrm{CdCl}_{2}, \mathrm{HgF}_{2}$, and $\mathrm{HgCl}_{2}$. 
Table 1. Experimental and calculated molecular properties of $\mathrm{CdCl}_{2}, \mathrm{HgF}_{2}$, and $\mathrm{HgCl}_{2}$.

\begin{tabular}{|c|c|c|c|c|}
\hline $\mathrm{XY}_{2}$ & & $\mathrm{CdCl}_{2}$ & $\mathrm{HgF}_{2}$ & $\mathrm{HgCl}_{2}$ \\
\hline \multicolumn{5}{|l|}{ Crystals } \\
\hline \multirow{2}{*}{\multicolumn{2}{|c|}{$\begin{array}{l}\text { Coordination number } \\
\text { of } \mathrm{X}\end{array}$}} & $5.002(10)$ & $4.53[17]$ & $4.378 / 4.393$ \\
\hline & & 6 & $8[17]$ & 2 \\
\hline$V_{z z}$ at $\mathrm{X}\left[10^{21} \mathrm{~V} / \mathrm{m}^{2}\right]$ & calc & $2.4(5)$ & - & $-71.8[5]$ \\
\hline$\eta$ at $\mathrm{X}$ & calc & $0.0(0)$ & - & $0.064[5]$ \\
\hline$V_{z z}$ at $\mathrm{X}\left[10^{21} \mathrm{~V} / \mathrm{m}^{2}\right]$ & $\exp$ & $\pm 2.2(4)[4]$ & - & $\pm 75.7[5]$ \\
\hline$\eta$ at $X$ & $\exp$ & $0.12(7)[4]$ & - & $0.065[5]$ \\
\hline \multicolumn{5}{|l|}{ Molecules } \\
\hline \multicolumn{4}{|c|}{$R_{\mathrm{X}-\mathrm{Y}}$ [a.u.] from total } & $4.23(3)$ \\
\hline$R_{\mathrm{X}-\mathrm{Y}}[$ a.u.] from force & calc & $4.22(3)$ & $3.64(2)$ & $4.23(3)$ \\
\hline$R_{\mathrm{X}-\mathrm{Y}}[$ a.u.] & $\exp$ & $4.21[18]$ & - & $4.29[18]$ \\
\hline \multirow[t]{2}{*}{$\omega_{\mathrm{sym}}\left[\mathrm{cm}^{-1}\right]$} & calc & 357 & 620 & 345 \\
\hline & $\exp$ & $329[9]$ & $588[10]$ & $346[9]$ \\
\hline \multirow[t]{2}{*}{$\omega_{\text {bend }}\left[\mathrm{cm}^{-1}\right]$} & calc & 88 & 164 & 100 \\
\hline & $\exp$ & 88.5 [9] & $171.5[10]$ & $107[9]$ \\
\hline$V_{z z}$ at $\mathrm{X}\left[10^{21} \mathrm{~V} / \mathrm{m}^{2}\right]$ & calc & $-35.0(2)$ & $-58.9(3)$ & $-73.3(5)$ \\
\hline$a_{1}$ & calc & $3.82(5)$ & $5.86(4)$ & $4.72(3)$ \\
\hline
\end{tabular}

The $V_{z z}$ values at the metal atoms were fitted with a function $a_{0} x^{-3}$, assuming a point charge like dependence and $a_{0} x^{-a_{1}}$ as predicted by Butz $[11,12]$, assuming a stronger dependence of $V_{z z}$ on the bond distance. The first function did not fit the results well, whereas the second function is adequate. The exponents $a_{1}$ are given in Table 1 .

\subsection{Bond Angles}

For the equilibrium distances given in Table 1 (using the force criterion) the bond angles were varied from $180^{\circ}$ to $80^{\circ}$. The resulting forces were split into their radial and tangential component, and the latter was used to calculate the bending vibrational frequencies given in Table 1. The agreement with experimental data taken from $[9,10]$ is excellent.

Furthermore, the contributions from different electronic states $(\mathrm{Cd}-4 \mathrm{p}, \mathrm{Cl}-3 \mathrm{~s}, \mathrm{Cd}-4 \mathrm{~d}$, and valence states for $\mathrm{CdCl}_{2} ; \mathrm{Hg}-5 \mathrm{p}, \mathrm{F}-2 \mathrm{~s}, \mathrm{Hg}-5 \mathrm{~d}$, and valence states for $\mathrm{HgF}_{2}$; and $\mathrm{Hg}-5 \mathrm{p}, \mathrm{Cl}-3 \mathrm{~s}, \mathrm{Hg}-5 \mathrm{~d}$, and valence states for $\mathrm{HgCl}_{2}$ ) to the EFG at the metal atoms were investigated. The results are presented in Fig. 5 in the form of Czjzek-plots (see e.g. [13]). Within a point charge model $V_{z z}, \eta$-trajectories of a three-atomic molecule would start in such a plot at the lower line for $\eta=0$ corresponding to a stretched molecule $\left(\theta=180^{\circ}\right)$, cross the line at $\eta=1$ at $2 \theta_{\text {magic }}=109.47^{\circ}$, get reflected at the upper line at $\eta=0$ for $\theta=90^{\circ}$, cross $\eta=1$ again at $\theta=180^{\circ}-2 \theta_{\text {magic }}$, and finally reach the starting point for $\theta=0^{\circ}$ again, as shown in the last plot of Figure 5. Note that $\theta_{\text {magic }}=54.74^{\circ}$ is the magic angle for which $3 \cos ^{2} \theta_{\text {magic }}-1=0$.
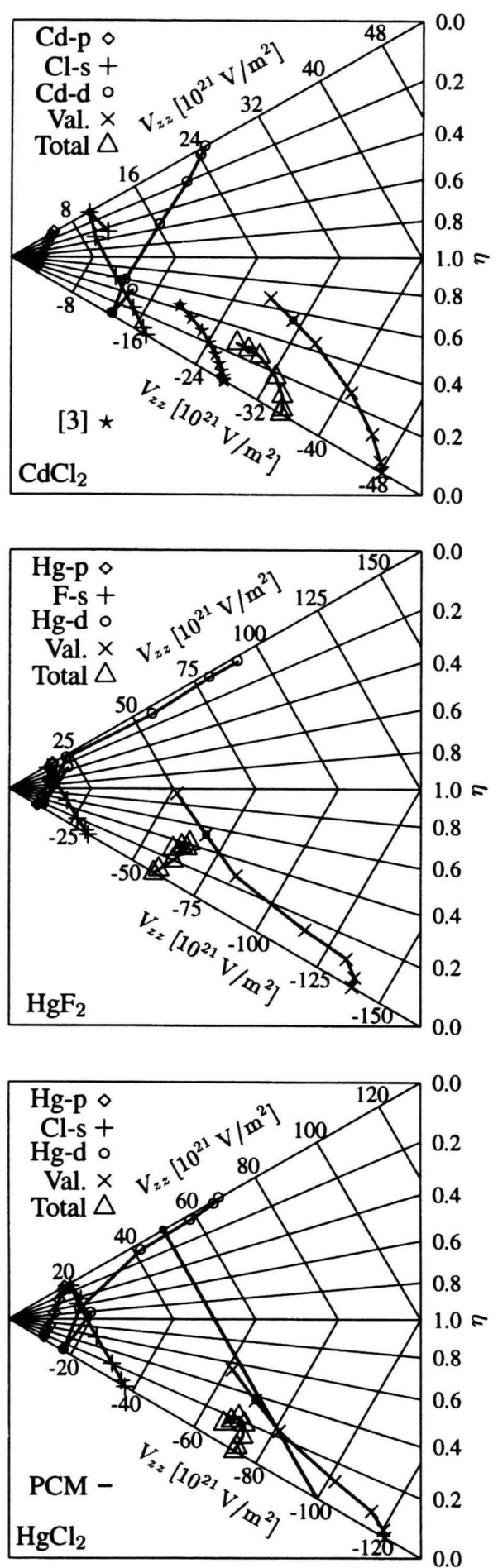

Fig. 5. Czjzek-plots of the contributions of different states to the metal EFG in $\mathrm{CdCl}_{2}, \mathrm{HgF}_{2}$, and $\mathrm{HgCl}_{2} . . \ldots \theta=90^{\circ}$. The first plot contains the data of Hemmingsen and Ryde [3], the last shows the predictions of the point charge model (PCM). 
Obviously, the EFGs from the metal p-(semicore), the $\mathrm{F} 2 \mathrm{~s}-$, the $\mathrm{Cl} 3 \mathrm{~s}-$, and the $\mathrm{Cd} 4 \mathrm{~d}$-states exhibit a point charge-like behaviour, whereas those from the $\mathrm{Hg} 5 \mathrm{~d}$ states, the valence states, and the total EFG do not at all. Furthermore, it can be seen that the different contributions have opposite signs and therefore partially cancel each other.

Figure 5 also contains the results of Hemmingsen and Ryde for $\mathrm{CdCl}_{2}$ [3]. They used a bond distance $R_{\mathrm{Cd}-\mathrm{Cl}}=4.734$ a.u. (the sum of the covalent radii of the atoms) which differs from the equilibrium atom distance calculated within the present work (Section 5.1).

\section{Results for Crystals}

In order to compare the free molecule with the crystal of the same compound, $\mathrm{CdCl}_{2}$ was calculated as a solid using the structure determined by Pauling and Hoard [14]. The space group is $\mathrm{R} 3 \mathrm{~m}$ with $\mathrm{Cd}$ at $(0,0,0)$ and $\mathrm{Cl}$ at $(u, u, u)$. The reported internal coordinate $u=0.25(1)$ was checked by minimizing the total energy and found to be $u=0.251$ (2), in excellent agreement with the X-ray value. The EFG at $\mathrm{Cd}$ sites exhibited a very strong dependence on $u$ and at the equilibrium geometry was determined to be $+2.4(5) \cdot 10^{21} \mathrm{~V} / \mathrm{m}^{2}$, in good agreement with the experimental value $\pm 2.2 \cdot 10^{21} \mathrm{~V} / \mathrm{m}^{2}$ found by Fraenzke and Butz [4]. Similar calculations and experiments on $\mathrm{HgCl}_{2}$ have been performed by Tröger et al. [5] and showed the same good agreement.

For comparison, all results and some relevant crystal structure data are summarized in Table 1.

\section{Discussion}

\subsection{Basis Set}

Figure 2 shows how the investigated physical properties converge with respect to $R K_{\max }$ and good convergence is found for $R K_{\max }>7$. The dependence of total energy can easily be interpreted in terms of the variational principle: The better the basis set, the lower the total energy. The negative value of the force $F$ on $\mathrm{Cl}$ at $R K_{\max }=6.0$ illustrates the importance of a good basis set: A poor basis set can cause qualitatively wrong results. In other calculations too (see e.g. [15]), we observed that the basis set had a stronger effect on the forces than on other properties (e.g. $V_{z z}$ ).

\subsection{Unit Cell Dimensions and Molecular Properties}

The convergence of molecular properties with respect to the unit cell dimensions and the related intermolecular distances is shown in Figure 3. A separation of about 10 a.u. ( $5 \AA$ ) between atoms belonging to different cells is sufficient to make the spurious interactions negligible. Furthermore, molecular properties are calculated without additional information (e.g. on bond distances). Bond distances can be obtained using two different numerical approaches, namely from the total energy or directly using the forces at the nuclei. Usually these two methods yield almost identical results and the small discrepancies (e.g. in the case of $\mathrm{CdCl}_{2}$ ) as related to numerical reasons due to the truncated basis set.

The quadratic (linear) dependence of the total energy (force) is expected due to the expansion of the potentials (nuclear part, separated from electronic part) near the equilibrium distances up to harmonic terms. Nevertheless, even in the small range presented here higher order terms are visible.

The excellent agreement with experimental values for the derived bond distances, stretch vibrational as well as the bending vibrational frequencies shows that the FPLAPW method is applicable to molecules, too, but one must admit that a larger computational effort is required than for solids.

\subsection{Electric Field Gradients - Crystals and Molecules}

The good agreement between experimental and theoretical values for the internal coordinate $u$ and the EFG component $V_{z z}$ at $\mathrm{Cd}$ for crystalline $\mathrm{CdCl}_{2}$ is not surprising since the FPLAPW method was successfully used in this field before. Unfortunately, no experimental values of the EFGs in the molecules are available. Since the EFG is very sensitive to details in the asymmetry of the electron density, especially near the nucleus, this would be a further and very interesting test of the method applied to molecules.

Comparing the EFGs in crystals and molecules, we note a large difference in the case of $\mathrm{CdCl}_{2}$, whereas it is small for $\mathrm{HgCl}_{2}$. This can be explained by looking at the crystal structure: $\mathrm{Cd}$ is six-fold coordinated in the crystal, leading to an averaging over the EFG components from the respective neighbours, but two-fold coordinated in the molecule. For $\mathrm{HgCl}_{2}$, on the contrary, $\mathrm{Hg}$ is two-fold coordinated both in the crystal and the molecule. Figure 6 clearly shows that the $\mathrm{HgCl}_{2}$ crystal is composed of relatively isolated molecules and thus can be considered a molecular crystal, whereas $\mathrm{CdCl}_{2}$ clear- 

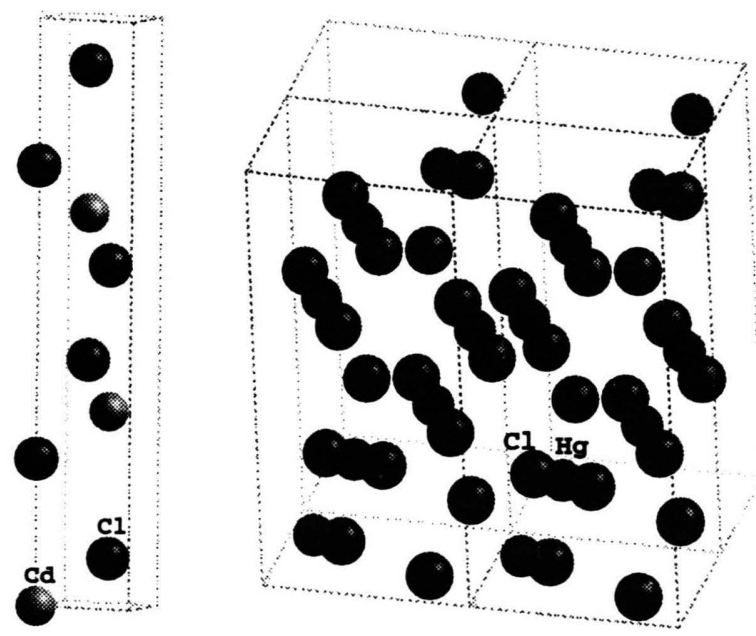

Fig. 6. Crystal structure of $\mathrm{CdCl}_{2}$ ([14], left) and $\mathrm{HgCl}_{2}$ ([19], right).

ly is an ionic crystal. Therefore the difference in EFG for $\mathrm{CdCl}_{2}$ between crystal and molecule is by far stronger than for $\mathrm{HgCl}_{2}$. For $\mathrm{HgF}_{2}$, however, a similar behaviour to that of $\mathrm{CdCl}_{2}$ can be expected due to the eight-fold coordination of $\mathrm{Hg}$ in the crystalline case.

\subsection{Electric Field Gradients - \\ Dependence on Intramolecular Quantities}

The dependence of EFGs at the metal atoms of the molecules exhibited no pure $r^{-3}$ behaviour, in contrast to the predictions of a point charge model. The $r^{-3}$-dependence of the point charge model is equivalent to a scaling down of the electron density with decreasing bond distance. Obviously, the dependence is much stronger and could be fitted with $r^{-a_{1}}, a_{1}>3$. An ideal ionic bond would give $a_{1}=3$. For $\mathrm{CdCl}_{2}$, the lowest value of $a_{1}$ was found. This is an indication that this bond is the most ionic one of the three investigated.

A more detailed discussion of the volume dependence of the EFG can be found in $[11,12]$.

The results for the bending of the molecules can be interpreted as follows: The energetically low lying electronic states behave point charge-like, whereas the energetically high (valence) states do not. This means that the semicore states are polarized by external charges but do not participate in bonding, whereas the valence states change their shape while bending the molecule due to changes in chemical bonding. It is interesting to note that, although attached to the same $\mathrm{Cl}$ ligand, the $\mathrm{Cd}-4 \mathrm{~d}$ states behave point charge like whereas the $\mathrm{Hg}-5 \mathrm{~d}$ states do not.
This reflects the fact described e.g. by Orgel [16] that the $\mathrm{Hg}$-d states participate in the chemical bond by hybridization, but the Cd-d states do not. This hybridization is also responsible for the preference of a linear, two-fold coordination of $\mathrm{Hg}$ and a more covalent bond, visible e.g. in the crystal structure of $\mathrm{HgCl}_{2}$ and in the higher resistance against bending of the investigated $\mathrm{Hg}$-compounds, whereas $\mathrm{Cd}$ prefers higher coordination numbers and a more ionic bond, in agreement with the discussion of the exponent $a_{1}$ (see above).

The EFG-contributions plotted in Fig. 5 arise only from electron densities within the metal atom sphere. The contributions from interstitial regions and from the other spheres are negligibly small.

The largest contribution arises from the valence states. This is in agreement with a corresponding statement of Tröger et al. for crystalline $\mathrm{HgCl}_{2}$ [5]: Contributions from the $\mathrm{Hg}-6 \mathrm{p}$ states $^{3}$ dominate the EFG. These states are almost unoccupied, but they contain the orthogonalized tail of the halogen wave functions [5], and therefore are called "off-site" contributions. The contributions of the deeper electronic states are smaller but by far not negligible. They partially cancel each other: The contributions of states localized at the metal site (metal-p and metal-d states) are positive, the others are negative. Therefore these contributions have also to be known as accurately as possible.

A comparison of the total EFG at $\mathrm{Cd}$ in $\mathrm{CdCl}_{2}$ with the results of Hemmingsen and Ryde [3] shows partial agreement in trend and in value. The difference in value can partly be caused by different bond distances: The larger bond distance of Hemmingsen and Ryde causes a lower $V_{z z}$ value. We consider our bond distance as more realistic because it was determined by minimizing the total energy, whereas Hemmingsen and Ryde added the covalent radii of the atoms only. When we rescale our trajectories to the bond distance of Hemmingsen and Ryde we obtain an excellent agreement to their results for the EFG-contribution from the valence states, whereas the total EFG itself does not agree as well. This could be a hint that energetically lower states are treated in adequately within the basis set of Hemmingsen and Ryde. The FPLAPW basis set has proven to describe the energetically lower states adequately in a lot of crystal calculations, as was also demonstrated in the present work. However, a direct experimental test for the calculated isolated molecules is not available yet.

\footnotetext{
3 These states are not occupied in the isolated atoms but become slightly occupied due to the chemical bonding.
} 


\section{Summary: Applicability of the FPLAPW Method to Molecules}

The results pesented in this work are an excellent proof of the applicability of the FPLAPW method to isolated molecules. It was shown that the calculation of molecular properties converges by increasing the distance between periodically repeated molecules. Furthermore, the experimentally available data (bond distances, stretchvibrational and bending-vibrational frequencies) are in good agreement with the calculated values.

Unfortunately, there are some serious drawbacks in applying the FPLAPW method to isolated molecules. Large unit cells are necessary to separate atoms belonging to different cells by at least 10 a.u. This requires an extended plane wave basis set (with a matrix size of more than 2000 for the small compounds investigated in this work) and therefore large main memory capacity and a lot of

[1] P. Blaha, K. Schwarz, P. Sorantin, and S. B. Trickey, Comput. Phys. Commun. 59(2), 399 (1990). Updated WIEN95 version was used.

[2] P. Blaha, P. Dufek, K. Schwarz, and H. Haas, Hyp. Int. 97/98, 3 (1996).

[3] L. Hemmingsen and U. Ryde, to be submitted to J. Phys. Chem. 1995.

[4] Th. Fraenzke and T. Butz, Z. Naturforsch. 74a, 89 (1992).

[5] W. Tröger, T. Butz, P. Blaha, and K. Schwarz, Hyp. Int. 80, 1109 (1993).

[6] J. P. Perdew, J. A. Chevary, S. H. Vosko, K. A. Jackson, M. R. Pederson, D. J. Singh, and C. Fiolhais, Phys. Rev. B 46(11), 6671 (1992).

[7] P. Blaha, K. Schwarz, P. Dufek, and R. Augustyn, WIEN95 - A Full Potential Linearized Augmented Plane Wave Package for Calculating Crystal Properties. Computational Quantum Chemistry Group, Technical University Vienna, Vienna 1995.

[8] T. Soldner, Elektrische Feldgradienten in Mercaptiden, Cadmium- und Quecksilberhalogeniden: Theorie und Experiment, Diplomarbeit, Universität Leipzig, 1996. computing time. This could prevent a useful application of the FPLAPW method for more complicated molecules.

However, since the FPLAPW basis set is excellently suited for EFG calculations and well tested in many crystal applications, calculations of the presented type help to check the EFG values obtained from molecular methods and to improve the basis sets used in these methods.

\section{Acknowledgement}

One of us (T. S.) wishes to thank Dr. Birkenheuer, Technische Universität München, for helpful discussions on vibrational frequencies. We further thank Dr. Dan, Universität Düsseldorf, for helping us with literature on vibrational spectra.

This work was partially supported by the DFG (grant Bu 594/9-1).

[9] A. Loewenschuss, A. Ron, and O. Schneoo, J. Chem. Phys. 50, 2502 (1969).

[10] A. Givan and A. Loewenschuss, J. Chem. Phys. 72, 3809 (1980).

[11] T. Butz, Physica Scripta 17, 87 (1978).

[12] T. Butz, Physica Scripta 17, 445 (1978)

[13] T. Butz, M. Ceolín, P. Ganal, P. Schmidt, M. A. Taylor, and W. Tröger, Physica Scripta 54, 234 (1996).

[14] L. Pauling and J. L. Hoard, Z. Kristallogr. 74, 546 (1930).

[15] T. Soldner, W. Tröger, T. Butz, P. Blaha, and K. Schwarz, Z. Naturforsch. A 53a, 404 (1998).

[16] L. E. Orgel, J. Chem. Soc. (1958) p. 4186.

[17] F. Ebert and H. Woitinek, Z. Anorgan. Allgem. Chem. 210, 269 (1933).

[18] P. Debye Jr. et al., Atom- und Molekularphysik. In A. Eucken and K.-H. Hellwege (eds.), Landold-Börnstein, Zahlenwerte und Funktionen aus Physik, Chemie, Astronomie, Geophysik und Technik. $6^{\text {th }}$ Ed. Springer, Berlin 1951.

[19] R. W. G. Wyckoff, Crystal Structures, Vol. 1, Wiley, New York 1963. 